\title{
Algo podría cambiar en el tratamiento de la apendicitis aguda no complicada
} Something may change in the management of uncomplicated acute appendicitis

El $7 \%$ de los seres humanos tendrá una apendicitis aguda (AA) en el trascurso de su vida. ${ }^{1}$ Desde hace 130 años, la apendicectomía es la cirugía de urgencia más frecuente..$^{1-2}$ Solo ante un plastrón o masa apendicular, se prefiere el tratamiento antibiótico a la cirugía inmediata.

En la apendicitis aguda no complicada (AANC), la inflamación se limita al apéndice cuya pared está íntegra. El paciente está en buen estado, con dolor y signos característicos en la fosa ilíaca derecha. En la ecografía, el apéndice no es compresible y su diámetro es mayor de $6 \mathrm{~mm}$. La grasa mesentérica es hiperecogénica y puede haber escaso líquido claro periapendicular o retrovesical. En la AA complicada, se deteriora el estado general y hay evidencias de perforación, absceso periapendicular o peritonitis generalizada. No se discute la cirugía en la AA complicada.

En la última década, se propuso el tratamiento médico de la AANC para disminuir los costos del alto número de apendicectomías y evitar el estrés quirúrgico y las complicaciones de la cirugía con anestesia general, que ocurrían en el 7-17 \% de los casos. ${ }^{1-4}$ Se han publicado metaanálisis de series de adultos con AANC, tratados exclusivamente con antibióticos, con resultados disímiles: fueron efectivos en el 68-95\% de los casos y la tasa de recurrencia de AA varió entre el $4,4 \%$ y el $39 \%$. . $^{3-6}$ En un metaanálisis realizado en 2016 sobre 5 series que sumaron 1430 adultos con AANC, la efectividad del tratamiento médico fue del $63 \%$. Sin embargo, el 53-79 \% de los pacientes fue finalmente operado. Estos estudios tienen baja calidad de evidencia sobre el resultado terapéutico, por defectos en el diseño. ${ }^{7}$

En 2017, un metaanálisis seleccionó 10 estudios en los que se analizaba el resultado del tratamiento médico y de la cirugía de la AANC en un total de 413 niños. El tratamiento antibiótico fue efectivo en el $97 \%$ de los pacientes y evitó la cirugía en el $82 \%$. Tanto las complicaciones como el tiempo de internación fueron similares. La recurrencia de AA luego del tratamiento médico fue del $14 \% .{ }^{1}$ Solo 6 de estos estudios fueron comparativos y uno solo fue aleatorio, pero con insuficiente número de casos. $^{2}$ Este estudio piloto permitió saber que el tratamiento antibiótico de la AANC ofrecía una tasa aceptable de complicaciones o de recurrencia de AA y, además, que la apendicectomía tardía, por falla del tratamiento médico, no tenía más complicaciones que la cirugía inmediata. ${ }^{2,46}$

Hasta 2018, no hay ningún estudio en niños, con fortaleza estadística suficiente, para afirmar que el tratamiento antibiótico de la AANC es mejor que la cirugía. ${ }^{1,4,6}$

En 2017, comenzó un estudio aleatorio, multicéntrico, que siguiendo protocolos estadísticos y rigurosos aspectos éticos; tiene como objetivo comparar los resultados del tratamiento médico y de la cirugía inmediata en la AANC. En este estudio, conocido como Appy Trial, se planea reclutar a 978 niños con AANC de 5 a 16 años de edad y seguirlos durante 1 año luego del episodio inicial. ${ }^{6}$ El diagnóstico de AANC será clínico y ecográfico, y se empleará la tomografía axial computada (TAC) con contraste oral solo cuando la clínica o la ecografía sean dudosas. Se excluirán los pacientes con sospecha de perforación, los que hubieran recibido más de una dosis de antibiótico y los menores de 5 años. Los niños pequeños tienen una evolución rápida de la AA, su presentación puede ser atípica y, en el 12,5-30\% de los casos, tienen una perforación apendicular inicial. ${ }^{6,8,9}$ Tampoco se incluirán niños con antecedentes compatibles con otros episodios de AA, los que tengan mucoviscidosis o enfermedades malignas y las adolescentes embarazadas. La presencia de un fecalito apendicular no excluirá al niño del estudio. ${ }^{6}$ Algunos autores desaconsejan el tratamiento médico en estos casos, por mayor incidencia de complicaciones. ${ }^{2-4}$

En los niños con AANC elegibles, se asignará aleatoriamente el tratamiento antibiótico elegido por cada centro o la cirugía videolaparoscópica inmediata como mejor opción quirúrgica. ${ }^{6} \mathrm{El}$ tratamiento antibiótico será intravenoso por un mínimo de $12 \mathrm{~h}$, y se controlará su resultado a las 24 y a las 48 h. Ante una buena respuesta, se completarán 10 días por vía oral. Si hay empeoramiento a las $24 \mathrm{~h}$ o falta de mejoría a las $48 \mathrm{~h}$ del inicio, se indicará la cirugía.

Se obtendrá el consentimiento para asignar aleatoriamente el tratamiento al niño con AANC. 
En el estudio piloto, preparatorio del Appy Trial, solo el $40 \%$ de los padres de los niños elegibles aceptó el proceso aleatorio. ${ }^{2}$ Otro estudio en niños con AANC no pudo realizarse en forma aleatoria y, aunque comunicaba un $98,7 \%$ de efectividad del tratamiento médico, no tenía fortaleza estadística. ${ }^{3}$

Si el Appy Trial demostrara que el tratamiento antibiótico de la AANC es más conveniente que la operación inmediata, será una revolución en la cirugía pediátrica.

\section{Esto merece algunas reflexiones:}

El cirujano pediatra realizaría el diagnóstico de la AANC, patología frecuente en su práctica diaria, y solo operaría cuando fallara el tratamiento antibiótico. ${ }^{4} \mathrm{Si}$ el diagnóstico estuviera a cargo del clínico pediatra, sería conflictivo. El diagnóstico de la AA es, muchas veces, difícil y hay retrasos en el $27-57 \%$ de los casos. En centros del mayor nivel, en el 4-6\% de las apendicectomías, el apéndice estaba sano. ${ }^{6,9}$

En la AA, la ecografía tiene una sensibilidad del $99 \%$ y una especificidad del $95 \%$. Solo en casos dudosos, se utiliza la TAC con contraste oral, que tampoco es infalible y puede omitir una perforación apendicular.

Cuando no se opera a un niño con una "supuesta" AANC, podría indicarse un tratamiento antibiótico innecesario si tuviera una adenitis mesentérica viral o una ruptura folicular ovárica. Asimismo, habría riesgo de no operar oportunamente a un niño con un divertículo de Meckel infectado o complicado o con otras causas quirúrgicas de infección intraperitoneal.

La AA es motivo frecuente de juicios por mala praxis médica. Se deberá validar tanto la conducta no quirúrgica en la AANC como la cirugía diferida $48 \mathrm{~h}$ cuando el tratamiento antibiótico sea inefectivo.

La medicina ejercida en forma "defensiva" motiva estudios innecesarios. La falta de una ecografía o una TAC, para tener más certeza diagnóstica de una AANC, podría motivar derivaciones inconvenientes de pacientes con una patología de baja complejidad a centros de alta complejidad.

$\mathrm{Si}$, en nuestro medio, se pretende disminuir los costos con el tratamiento médico de la AANC, el tiempo de internación debería ser similar al de una apendicectomía videolaparoscópica. Un estudio no aleatorio en niños con AANC mostró un tiempo promedio alto, de 5 días de internación, para el tratamiento antibiótico. ${ }^{8}$ En otro estudio japonés, la internación para el tratamiento médico de la AANC fue de 6 días porque el sistema de salud lo consideraba preferible. ${ }^{3}$ En cada caso, deberá decidirse si el nivel de alarma de la familia permite el alta precoz para completar el tratamiento ambulatoriamente.

No operar una AANC resulta atractivo para el niño y sus padres, pero hay incertidumbre sobre la efectividad del tratamiento médico. En un estudio, 82 de los 197 pacientes con AANC no respondió al antibiótico y fueron operados (el $42 \%) .{ }^{8}$ Superado el episodio inicial, persistirá el temor a una recurrencia, especialmente, en pacientes del área rural o los que viajen por vacaciones, por estudio, por campamentos, por giras deportivas, etc. Un estudio mostró la recurrencia de la AANC en el $28 \%$ de los pacientes tratados con antibióticos. ${ }^{3}$ Fue publicado que más del $40 \%$ de los niños con AANC tratados con antibióticos tuvieron reinternaciones y fueron, al final, operados. ${ }^{8,10}$

Es necesario demostrar estadísticamente que el tratamiento médico de la AANC en los niños mayores de 5 años es más efectivo que la cirugía inmediata, con menos complicaciones y baja recurrencia. Se deben definir los criterios para seleccionar a los pacientes. ${ }^{6}$

Hasta conocer los resultados que apoyen la elección del tratamiento médico en la AANC, no deberíamos cambiar la actual indicación de cirugía precoz. ${ }^{2,4,6}$

Dr. Fernando L. Heinen Especialista en Cirugía Infantil Sanatorio de Los Arcos (SMG) Ciudad de Buenos Aires fernando.heinen@gmail.com

http:/ / dx.doi.org/10.5546/ aap.2019.68

Texto completo en inglés:

http: / / dx.doi.org/10.5546/ aap.2019.eng.68

Cómo citar: Heinen FL. Algo podría cambiar en el tratamiento de la apendicitis aguda no complicada. Arch Argent Pediatr 2019;117(2):68-70.

\section{REFERENCIAS}

1. Georgiou R, EatonS, Stanton MP, Pierro A, etal.Efficacy and Safety of Nonoperative Treatment for Acute Appendicitis: A Meta-analysis. Pediatrics. 2017; 139(3):e20163003.

2. Svensson JF, Patkova B, Almstrom M, Naji H, et al. Nonoperative treatment with antibiotics versus surgery for acute nonperforated appendicitis in children: a pilot randomized controlled trial. Ann Surg. 2015; 261(1):67-7.1

3. Tanaka Y, Uchida H, Kawashima H, Fujiogi M, et al. 
Long-term outcomes of operative versus nonoperative treatment for uncomplicated appendicitis. J Pediatr Surg. 2015; 50(11):1893-7.

4. Gorter R, Van der Lee JH, Heijsters FA, Cense HA, et al Outcome of initially nonoperative treatment for acute simple appendicitis in children. J Pediatr Surg. 2018; 53(9):1849-54.

5. Mason RJ, Moazzez A, Sohn H, Katkhouda N. Metaanalysis of randomized trials comparing antibiotic therapy with appendectomy for acute uncomplicated (no abscess or phlegmon) appendicitis. Surg Infect (Larchmt). 2012; 13(2):74-84.

6. Hall NJ, Eaton S, AbboO, Arnaud AP, et al. Appendectomy versus non-operative treatment for acute uncomplicated appendicitis in children: study protocol for a multicentre, open-label, non-inferiority, randomised controlled trial. BMJ Paediatr Open. 2017; 1(1):e000028.

7. Rollins KE, Varadhan KK, Neal KR, Lobo DN. Antibiotics versus appendicectomy for the treatment of uncomplicated acute appendicitis: an updated meta-analysis of randomised controlled trials. World J Surg. 2016; 40(10):2305-18.

8. Caruso AM, Pane A, Garau R, Atzori P, et al. Acute appendicitis in children: not only surgical treatment. $J$ Pediatr Surg. 2017; 52(3):444-8.

9. Marzuillo P, Germani C, Krauss BS, Barbi E. Appendicitis in children less than five years old: a challenge for the general practitioner. World J Clin Pediatr. 2015; 4(2):19-24.

10. Bachur RG, Lipsett SC, Monuteaux MC. Outcomes of Nonoperative Management of Uncomplicated Appendicitis. Pediatrics. 2017; 140(1):e20170048.

\section{Chupete: Asesorar en lugar de prohibir. La nueva Iniciativa del Hospital Amigo del Niño Pacifiers: Counseling instead of prohibiting. The new Baby-friendly Hospital Initiative}

\section{El inicio}

Las primeras horas y días de la vida de un recién nacido son un periodo crítico para establecer la lactancia y para proporcionar a las madres el apoyo que necesitan para amamantar exitosamente a sus hijos. Se considera que la leche humana es la norma biológica para alimentar al bebé y una acción preventiva tanto para la madre (cáncer) como para el niño (infecciones).

La Declaración Conjunta OMS / Unicef (Ginebra, 1989) instó a todos los servicios de maternidad y atención de recién nacidos a poner en marcha las medidas que se resumen en los "Diez pasos hacia una feliz lactancia natural". ${ }^{1}$

Un año después, la "Declaración de Innocenti", producto de una reunión de la OMS / UNICEF realizada en el Ospedale degli Innocenti, en Florencia, Italia, realizó un fuerte llamamiento a los gobiernos del mundo para apoyar la lactancia materna a través de programas y legislaciones, por ejemplo, el derecho a amamantar de la mujer que trabaja y jerarquizó la implementación de los "Diez pasos para una lactancia exitosa". ${ }^{2}$

\section{Iniciativa Hospital Amigo del Niño}

Ambas declaraciones pueden tomarse como los antecedentes inmediatos de la Iniciativa Hospital Amigo del Niño (IHAN). Esta iniciativa establece que los Diez pasos para una feliz lactancia natural son de cumplimiento obligado en los hospitales acreditados por Unicef como
Hospital Amigo del Niño.

En la actualidad se estima que solo el $10 \%$ de los nacimientos a nivel mundial se producen en hospitales galardonados por la IHAN como Hospital Amigo del Niño. ${ }^{3}$ Si bien se pudo demostrar que el programa beneficia la lactancia de las madres con bajo nivel de educación, ${ }^{4}$ ha recibido críticas en cuanto a las dificultades de sustentabilidad. Asimismo, se cuestionó la imposición vertical de las recomendaciones. Estas apreciaciones fueron expresadas en el Sumario Ejecutivo de la nueva Guía de Implementación de la IHAN. ${ }^{3}$

Personalmente, sostengo que en la redacción original del Paso 9, "No dar a los niños alimentados a pecho ni biberones ni chupetes", prevalece el espíritu fundamentalista sobre el científico, adoptado por ciertos grupos y/o asociaciones profesionales que han logrado institucionalizar una conducta intransigente y estricta con respecto al amamantamiento que se cristalizó en todo el ámbito de la niñez.

Esta postura tuvo su fundamento en un momento en que el porcentaje de niños alimentados con pecho exclusivo era ínfimo con la consecuencia de una elevada mortalidad y morbilidad infantil (alteración de la microbiota y contaminación de la leche). De esta manera la lucha en favor de la lactancia se constituyó en una medida salvadora de vidas.

La oposición al uso del chupete está basada 